\title{
High RhoA expression at the tumor front in clinically localized prostate cancer and association with poor tumor differentiation
}

\author{
WEIHUA CHEN $^{1,2}$, NICOLAS BARRY DELONGCHAMPS ${ }^{3}$, KAILI MAO ${ }^{1}$, FRÉDÉRIC BEUVON ${ }^{4}$, \\ MICHAËL PEYROMAURE ${ }^{3}$, ZHONGMIN LIU $^{5}$ and ANH TUAN DINH-XUAN ${ }^{2,5}$
}

\footnotetext{
${ }^{1}$ Department of Urology, Shanghai East Hospital, Tongji University School of Medicine, Shanghai 200120, P.R. China;

${ }^{2}$ Department of Functional Physiology, School of Medicine, Cochin Hospital, Paris Descartes University; Departments of ${ }^{3}$ Urology and ${ }^{4}$ Pathology, Cochin Hospital, Paris Descartes University, Paris 75014, France;

${ }^{5}$ Clinical and Translational Research Center, Shanghai East Hospital, Tongji University School of Medicine,
} Shanghai 200120, P.R. China

Received October 26, 2014; Accepted August 20, 2015

DOI: $10.3892 / \mathrm{ol} .2015 .4070$

\begin{abstract}
Ras homolog gene family, member A (RhoA) has been reported as essential to the invasion process and aggressiveness of numerous cancers. However, there are only sparse data on the expression and activity of RhoA in clinically localised prostate cancer. In numerous cancers, tumour cells at the invasive front demonstrate more aggressive behaviour in comparison with the cells in the central regions. In the present study, the expression and activity of RhoA was evaluated in 34 paraffin-embedded and 20 frozen prostate tissue specimens obtained from 45 patients treated with radical prostatectomy for clinically localised cancer. The expression patterns of RhoA were assessed by immunohistochemical staining and western blotting. Additional comparisons were performed between the tumour centre, tumour front and distant peritumoural tissue. RhoA activity was assessed by G-LISA. Associations between RhoA expression and the clinical features and outcome of the patients were also analysed. The present study found an increasing gradient of expression from the centre to the periphery of index tumour foci. RhoA expression was significantly increased at the tumour front compared to the tumour centre, which was determined using immunohistochemistry $(\mathrm{P}=0.001)$. Increased RhoA expression was associated with poor tumour differentiation in the tumour front $(\mathrm{P}=0.044)$ and
\end{abstract}

Correspondence to: Professor Anh Tuan Dinh-Xuan, Department of Functional Physiology, School of Medicine, Cochin Hospital, Paris Descartes University, 27 Faubourg Saint-Jacques Road, Paris 75014, France

E-mail: anh-tuan.dinh-xuan@cch.aphp.fr

Professor Zhongmin Liu, Clinical and Translational Research Center, Shanghai East Hospital, Tongji University School of Medicine, 150 Jimo Road, Shanghai 200120, P.R. China

E-mail: zhongmin_liu@sina.com

Key words: Ras homolog gene family, member A, prostate cancer, tumour front, tumour differentiation, invasive phenotype tumour centre $(\mathrm{P}=0.039)$. Subsequent to a median follow-up period of 52 months, the rate of prostate-specific antigen (PSA) relapse was increased in patients with higher RhoA expression at the tumour front when compared with patients with lower RhoA expression (62.5 vs. 35.0\%), although the difference was not significant $(\mathrm{P}=0.09)$. There was no association between RhoA expression and the PSA level or pathological stage in the present study. In conclusion, RhoA expression was increased at the tumour front and was associated with poor tumour differentiation in the tumour front and tumour centre, indicating the potential role of RhoA in prostate cancer. RhoA expression may also act as a prognostic factor in prostate cancer. The present data provide a foundation for novel therapeutic approaches by targeting RhoA in prostate cancer.

\section{Introduction}

Prostate cancer is the most prevalent malignancy and the second leading cause of cancer-associated mortality in men in Western countries (1). Several autopsy studies have revealed, however, that the majority of prostate cancers remain latent (2-4). These small and well-differentiated cancers, termed clinically insignificant (5), may not affect the health of patients. If these lesions are detected by prostate-specific antigen (PSA) screening and an extended biopsy, it is recommended that the tumours not be treated with radical treatments or treatments that lead to morbidity (6), but be managed by active surveillance. Distinguishing these latent cancers from aggressive cancers, which may spread and develop distant metastasis, remains a critical issue.

The molecular mechanisms that differentiate cancers with an invasive phenotype from those that remain latent within the gland continue to be largely unknown. Numerous factors have been implicated in the process of prostate cancer invasion and metastasis, but the initial molecular transforming events that occur in prostatic epithelial cells and the local microenvironment remain unknown $(7,8)$. The capacity of tumour cells to migrate and invade the prostate tissue is crucial in the initial steps of tumour progression (9). 
Ras homolog gene family, member A (RhoA) guanosine triphosphatase (GTPase) is a well-established regulator of the dynamic and spatiotemporal rearrangement of the cell cytoskeleton during cell migration (10). Previous studies have revealed that RhoA is active at the rear of cells and promotes tail retraction, and RhoA is also localised at the leading edge of migrating cells, where it regulates protrusion at the front of cells (10-12). RhoA promotes the invasive behaviour of tumour cells through invadopodia and bleb-driven amoeboid invasion (13-15). This has been demonstrated to be upregulated and participate in the tumour invasion process of numerous solid tumours, including ovarian carcinoma (16), ameloblastoma (17) and breast cancer (18).

Although a previous study has indicated that overexpression of RhoA is present in prostate cancer tissues (19), the activity of RhoA has been poorly studied in localised and locally advanced prostate cancer. Notably, the pattern of RhoA expression has not been evaluated in distinct tumours. Considering the potential role of RhoA in local tumour invasion, RhoA expression may differ throughout the prostate gland.

The aim of the present study was to evaluate and compare the expression and activity of RhoA in specimens obtained from radical prostatectomy (RP) performed on patients treated for localised or locally advanced prostate cancer, and to assess the value of RhoA as a prognostic marker of aggressiveness in prostate lesions. Three zones were compared, consisting of the tumour centre, the tumour front and peritumoural tissue.

\section{Patients and methods}

Patients. All human tissues were obtained from the Department of Surgical Pathology, Cochin Hospital (Paris, France), in accordance with the ethical policies and procedures of the Institutional Review Board of Cochin Hospital. All patients provided informed consent prior to participation in the present study. Details that may disclose the identity of the enrolled subjects have been omitted. Tumour tissues were graded according to the modified Gleason grading system (20). The pathological stage was determined according to the 2009 tumour-node-metastasis classification for prostate cancer (21).

In total, 34 patients diagnosed with clinically localised prostate cancer that had been treated with RP were enrolled in the present study. PSA relapse was experienced by 16 patients during the median follow-up duration of 52 months. Clinical data records included the patient age, PSA level, pathological stage, Gleason score and follow-up times.

Immunohistochemistry. Fresh-cut sections $(5-\mu \mathrm{m})$ were selected from formalin-fixed paraffin-embedded tissue blocks that contained tissue with the highest density of tumour cells and the highest Gleason scores, termed the index tumour. The sections were deparaffinised with xylene (Sigma-Aldrich, St. Louis, MO, USA) twice for $5 \mathrm{~min}$, rehydrated through an alcohol gradient, blocked with $0.3 \% \mathrm{H}_{2} \mathrm{O}_{2} /$ methanol (Sigma-Aldrich) at room temperature for $30 \mathrm{~min}$, and heated for $40 \mathrm{~min}$ at $96^{\circ} \mathrm{C}$ in $10 \mathrm{mM}$ sodium-citrate solution ( $\mathrm{pH} 6.0$; Sigma-Aldrich) for antigen retrieval. The slides were then incubated with goat anti-human polyclonal anti-RhoA antibody (dilution, 1:50; catalogue number, SC32039, Santa
Cruz Biotechnology, Inc., Dallas, TX, USA) overnight at $4^{\circ} \mathrm{C}$, and were then incubated with biotinylated rabbit anti-goat secondary antibody (dilution, 1:200; catalogue number, BA-5000; Vector Laboratories, Burlingame, CA, USA) at room temperature for $60 \mathrm{~min}$. This was followed by incubation with Vectastain Elite ABC Reagent (catalogue number, PK-7100; Vector Laboratories) for $30 \mathrm{~min}$. Peroxidase activity was examined using 3,3'-diaminobenzidine (catalogue number, D4418; Sigma-Aldrich). The sections were counterstained using hemalum and mounted using Permount (Thermo Fisher Scientific, Inc., Waltham, MA, USA).

Two pathologists independently scored the tissue staining for RhoA. The central region, which was fully composed of cancerous glands, was defined as the tumour centre. The edges of the tumour foci were termed the tumour front. Peritumoural benign prostate glands that did not exhibit intraepithelial prostatic neoplasia and were located on the same slide acted as the control, and were termed the peritumoural prostatic tissues. A semi-quantitative ordinal and categorical method (22) was used to evaluate staining across each region. The expression of RhoA was graded as follows: 0, no staining; 1, weak staining; 2, moderate staining; or 3, strong staining.

Western blot analysis. A total of 20 tissue samples were obtained from 11 patients that had undergone RP for the treatment of clinically localised or locally advanced prostate cancer. Immediately subsequent to surgical removal, small sections of tissue were dissected, snap-frozen and stored in liquid nitrogen. Histological analysis of the frozen sections was then performed. The percentage of tumour cells in each sample was evaluated. In total, 12 samples contained 50-90\% cancerous cells and were labelled as tumour tissues, and 8 samples containing no cancerous tissue were termed peritumoural prostatic tissue. These samples were then subjected to western blot analysis for RhoA expression and G-LISA analysis for RhoA activity. The proteins were extracted on ice with cold RIPA lysis buffer (catalogue number, 9806; Cell Signaling Technology, Inc., Danvers, MA, USA) containing a protease and phosphatase inhibitor (catalogue number, 88669; Thermo Fisher Scientific, Inc.).

Lysates were centrifuged at $12,000 \mathrm{x}$ g for $20 \mathrm{~min}$ at $4^{\circ} \mathrm{C}$, and the supernatant was collected. Total protein concentrations were determined using a bicinchoninic acid assay (BCA) protein-assay kit (catalogue number, 23250; Thermo Fisher Scientific, Inc.). Equal quantities of protein were subjected to $12 \%$ SDS-PAGE and then electrically transferred onto nitrocellulose membranes (Hybond-C; Amersham Biosciences, Uppsala, Sweden). The membranes were blocked for $1 \mathrm{~h}$ with $5 \%(\mathrm{w} / \mathrm{v})$ non-fat milk in phosphate-buffered saline with $0.1 \%$ (v/v) Tween-20 (PBST; Sigma-Aldrich), and incubated with polyclonal goat anti-human RhoA (dilution, 1:250; catalogue number, SC32039; Santa Cruz Biotechnology, Inc.) and monoclonal mouse anti-human $\beta$-actin (dilution, 1:500; catalogue number, SC47778; Santa Cruz Biotechnology, Inc.) primary antibodies overnight at $4^{\circ} \mathrm{C}$. Finally, the membranes were incubated with horseradish peroxidase (HRP)-conjugated secondary antibodies, consisting of the polyclonal rabbit anti-goat immunoglobulin G (IgG)-HRP antibody (dilution, 1:5,000; catalogue number, SC2768; Santa Cruz Biotechnology, Inc.) and polyclonal goat anti-mouse 

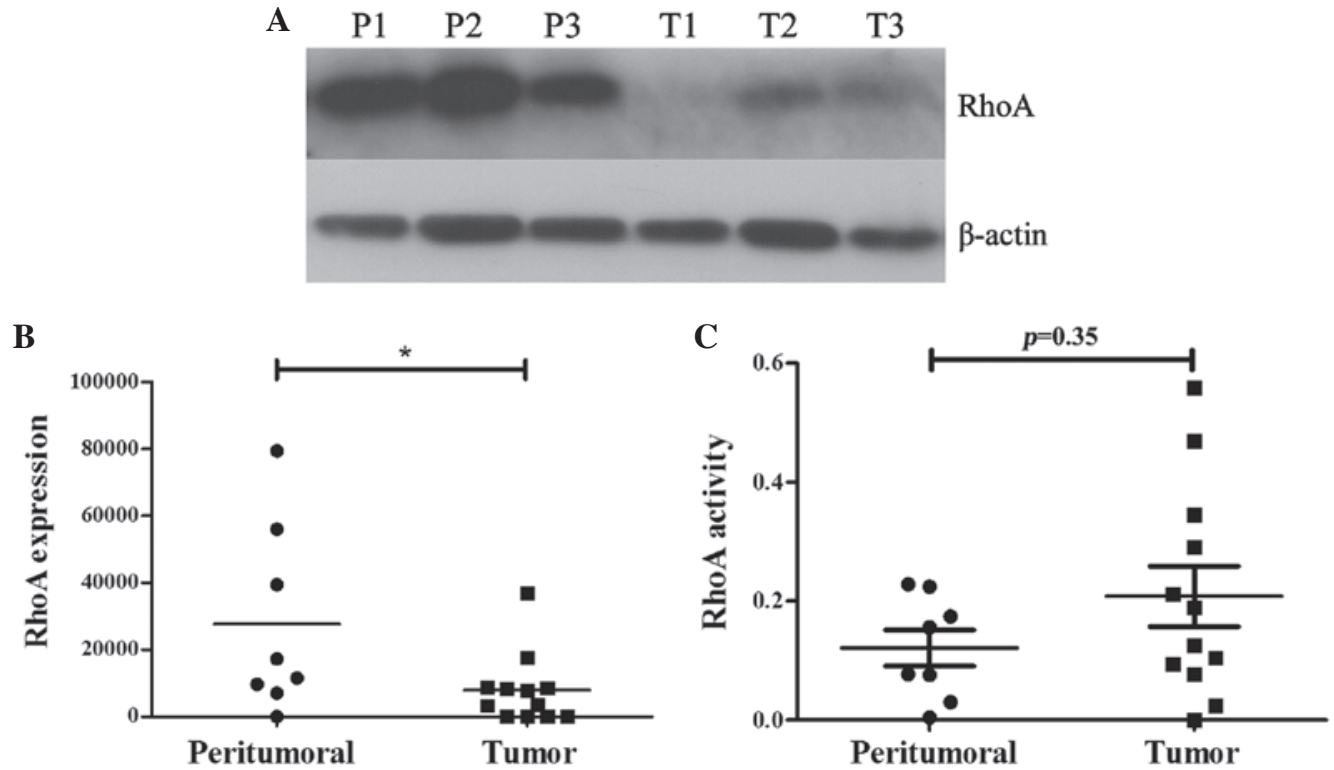

Figure 1. RhoA expression and activity in prostate cancer tissue specimens. (A) Representative western blots revealing RhoA expression in three prostate tumour tissue samples (T1-T3) and three peritumoral tissues (P1-P3). (B) Quantitative analysis of RhoA expression. The expression of RhoA in prostate cancer tissue specimens is significantly decreased compared with the peritumoral prostatic tissue samples ( $\mathrm{P}<0.05)$. (C) RhoA activity was increased in the tumour tissue samples compared with peritumoral prostatic tissues. However, this was not statistically significant $(\mathrm{P}=0.35)$. RhoA, Ras homolog gene family, member A.

IgG-HRP antibody (dilution, 1:5,000; catalogue number, SC2005; Santa Cruz Biotechnology, Inc.) for $1 \mathrm{~h}$ at room temperature.

Subsequent to washing three times with PBST, the proteins were visualised using an enhanced chemiluminescence Prime Western Blotting Detection kit (GE Healthcare Bio-Sciences, Pittsburgh, PA, USA). Images of the protein bands were captured using a digital imaging system (Image Quant LAS; GE Healthcare Bio-Sciences), and densitometric measurements of band intensity in the western blots were performed using ImageJ software (National Institutes of Health, Bethesda, MA, USA). The results are representative of three independent experiments.

G-LISA analysis. RhoA activity was assayed using a G-LISA RhoA Activation Assay Biochem kit (cat. no. BK124; Cytoskeleton, Inc., Denver, CO, USA), according to the manufacturer's instructions. Briefly, the prostate tissue samples were homogenised in ice-cold lysis buffer with a protease-inhibitor cocktail, and then centrifuged at $450 \mathrm{x} \mathrm{g}$ at $4^{\circ} \mathrm{C}$ for $1 \mathrm{~min}$. The supernatants were harvested and protein concentrations were measured using the Precision Red Advanced Protein Assay Reagent (catalogue number, ADV02; Cytoskeleton, Inc.) and were finally equalised with ice-cold lysis buffer to $1.0 \mathrm{mg} / \mathrm{ml}$. Equalised prostate-tissue protein extractions were transferred to a Rho-GTP-binding protein pre-coated plate (Cytoskeleton, Inc.). The plate was placed on an orbital microplate shaker (SSM1; Bibby Scientific Limited Group, Staffordshire, UK) at $0.72 \times \mathrm{g}$ for $30 \mathrm{~min}$ at $4^{\circ} \mathrm{C}$, and then incubated with monoclonal mouse anti-human anti-RhoA primary antibody (cat. no. GL01A; 1:250; Cytoskeleton, Inc.), followed by a polyclonal goat anti-mouse horseradish-conjugated secondary antibody (cat. no. GL02; 1:62.5; Cytoskeleton, Inc.), on an orbital microplate shaker (SSM1; Bibby Scientific Limited Group) at $0.72 \mathrm{xg}$ at room temperature, for $45 \mathrm{~min}$ each. The plate was then incubated with the HRP detection reagent at $37^{\circ} \mathrm{C}$ for $15 \mathrm{~min}$. Subsequent to the addition of HRP stop buffer, absorbance was read at $490 \mathrm{~nm}$ using an ELx808 Absorbance Microplate Reader (BioTek Instruments, Inc., Winooski, VT, USA).

Statistical analysis. Comparisons between data were performed using the $\chi^{2}$ test with a Yates continuity correction for independent samples, and the McNemar test was conducted for paired samples. Continuous data from two independent groups were compared using the Mann-Whitney $\mathrm{U}$ test. All tests performed were two tailed. $\mathrm{P}<0.05$ was considered to indicate a statistically significant difference.

\section{Results}

Patient characteristics. The clinicopathological features of the study population are summarised in Table I. A total of 34 patients were included in the IHC cohort and 11 patients were included in the WB and G-LISA cohort. The median age, median PSA level, pathological stages and Gleason scores in the two cohorts were comparable (Table I). The patients in the IHC cohort were followed up for a median duration of 52 months. A total of 16 (47.1\%) patients in the IHC cohort exhibited PSA relapses.

Expression and activity of RhoA in prostate tissues. RhoA expression was significantly decreased in prostate cancer tissue specimens compared with the peritumoural tissue specimens $(\mathrm{P}<0.05$; Fig. $1 \mathrm{~A}$ and $\mathrm{B})$. As the activation of RhoA is a prerequisite for the execution of its effects, the activity of RhoA was evaluated by G-LISA in the same tissue 


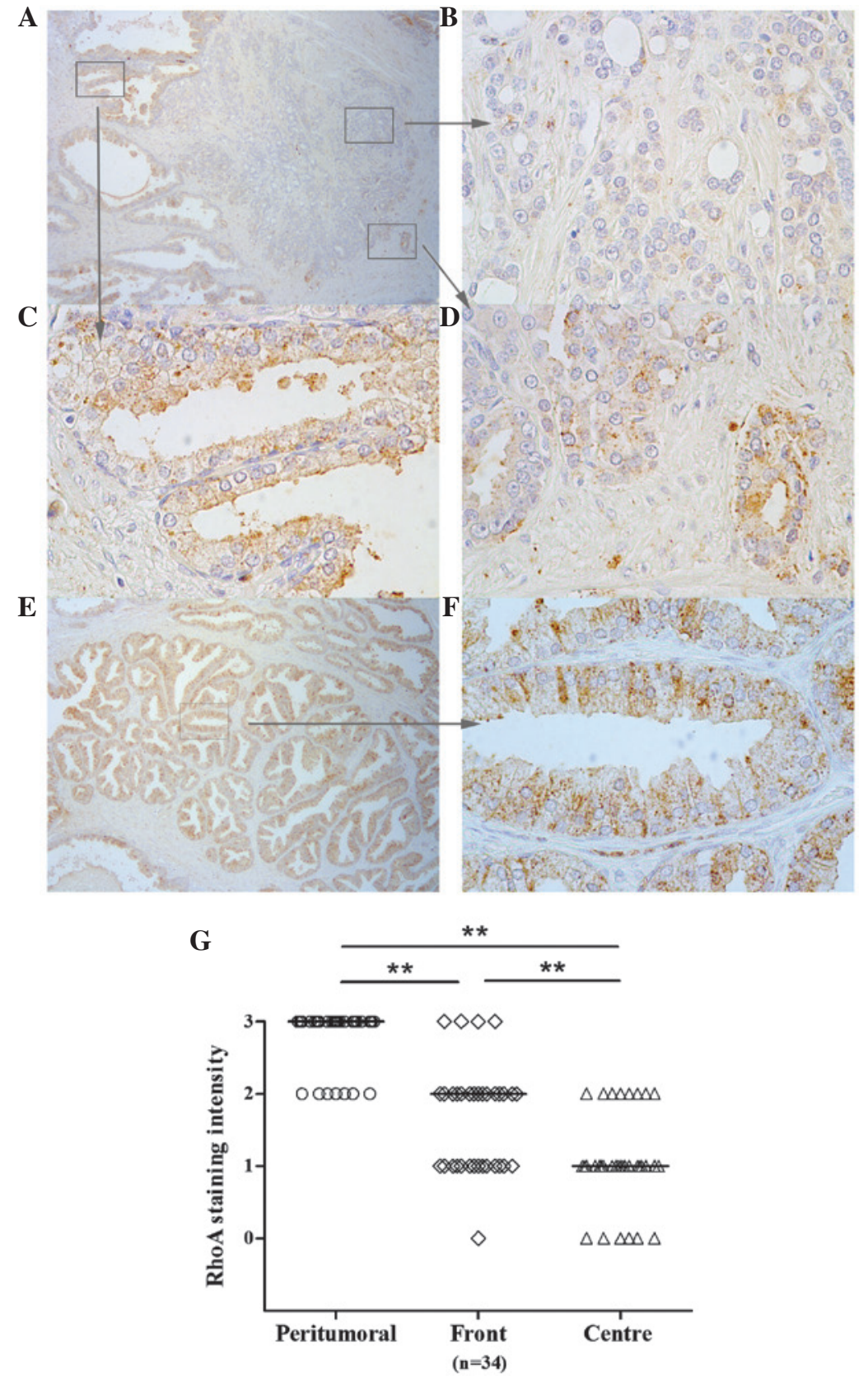

Figure 2. RhoA expression in prostate cancer, as assessed by immunohistochemistry. (A) An increasing gradient in the level of RhoA expression was identified between the tumour centre and the periphery of the index tumour foci. The expression of RhoA in (B) the prostate cancer tumour centre is significantly decreased compared with the expression at (D) the tumor front. However, $(\mathrm{C})$ the adjacent or (E and F) distal peritumoral prostate gland demonstrated the highest expression of RhoA. (G) Individual intensities of each sample and median intensities in each group. (A and E) Magnification, x50. (B-D and F) Magnification, $\mathrm{x} 400 .{ }^{* *} \mathrm{P}<0.01$. RhoA, Ras homolog gene family, member A.

samples. Although RhoA activity was increased in cancer tissues (optical density, 0.20) compared with non-cancer tissues (optical density, 0.12), the difference was not statistically significant $(\mathrm{P}=0.35)$.

Expression patterns of RhoA in cancerous prostate glands. To further evaluate the patterns of RhoA expression in prostate tissue specimens, immunohistochemistry was performed in 34 paraffin-embedded tissue specimens excised by RP. Analysis of the intensity of staining enabled the detection of various patterns of expression throughout the tissues. In the distant peritumoural tissue, immunohistological analysis revealed moderate or strong staining for RhoA expression in all tissues (Fig. 2). In the tumour centre, RhoA expression was significantly decreased, with moderate staining observed in only 8 tissues $(23 \%$; $\mathrm{P}<0.001)$. Strong staining was not observed in this region in any of the specimens. At the tumour front, staining was moderate or strong in 19 tissues (56\%), which was significantly increased compared with the incidence of moderate or strong staining in the centre of tumours (P<0.01; Fig. 2).

Association betweeen RhoA expression and adverse oncological features and outcomes. In the prostate cancer tissue specimens, RhoA expression was significantly increased in high-grade tumours that demonstrated a Gleason score 
Table I. Demographics of patients with prostate cancer and the characteristics of tissue specimens assessed by IHC or WB and G-LISA.

Cohort, n $(\%)$

\begin{tabular}{lcr} 
Characteristics & IHC & WB and G-LISA \\
\cline { 2 - 3 } Total & $34(100.0)$ & $11(100)$ \\
Median age, years (range) & $62(50-73)$ & $61(49-71)$ \\
Median PSA level, ng/ml (range) & $8.66(2.5-30)$ & $9.2(4.4-30)$ \\
Tumour stage & & $6(55)$ \\
T2 & $17(50.0)$ & $5(45)$ \\
T3 & $17(50.0)$ & $5(45)$ \\
Gleason score & & $3(27)$ \\
$3+3$ & $15(44.0)$ & $2(18)$ \\
$3+4$ & $11(32.0)$ & $1(9)$ \\
$4+3$ & $6(18.0)$ & N/A \\
$4+4$ & $2(6.0)$ & N/A \\
Median follow-up period, months (range) & $52(7-112)$ & $16(47.1)$ \\
PSA relapse & &
\end{tabular}

PSA relapse is defined as an increase in total serum PSA of $>0.2 \mathrm{ng} / \mathrm{ml}$ over two consecutive measurements. IHC, immunohistochemistry; WB, western blotting; PSA, prostate-specific antigen; N/A, not assessed.

A

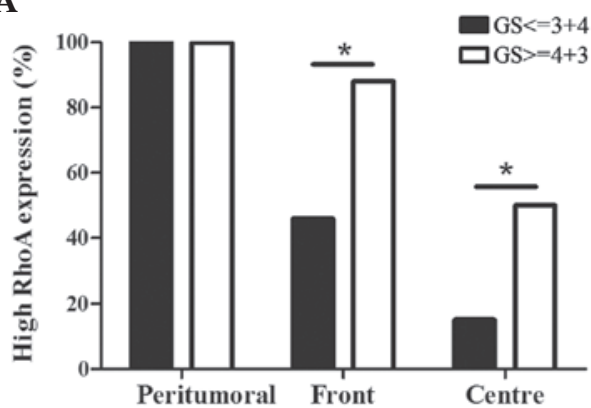

B

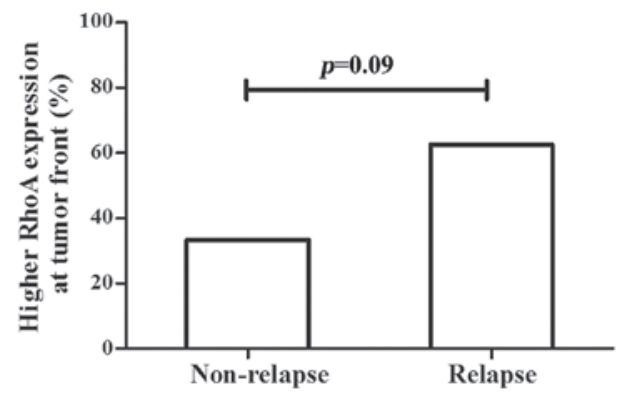

Figure 3. Association between the GS, PSA relapse and RhoA expression. (A) In the tumour centre and front, patients with poor tumor differentiation demonstrated a significantly increased expression of RhoA, as indicated by a GS of $4+3$ or higher and a percentage of RhoA staining score $\geq 2$ (tumour centre, 15 vs. $50 \%, \mathrm{P}=0.044$; tumour front, 46 vs. $88 \%, \mathrm{P}=0.039)$. ${ }^{*} \mathrm{P}<0.05$, high-grade differentiation vs. low-grade differentiation. (B) Patients that developed relapse possessed increased RhoA expression in tumour fronts compared to those that did not relapse. However, this difference was not statistically significant (62.5 vs. 35.0\%; P=0.089). RhoA, Ras homolog gene family, member A; PSA, prostate-specific antigen; GS, Gleason score.

$>3+4$, regardless of the location of the tumour cells, such as the tumour centre or front (Fig. 3A). Subsequent to a median follow-up duration of 52 months, $62.5 \%$ of the patients that experienced PSA relapse exhibited increased expression of RhoA at the tumour front compared with the expression in the tumour centre, while only $35 \%$ of patients without PSA relapse demonstrated increased RhoA expression at the tumour front (Fig. 3B; P=0.09). No association was identified between the RhoA expression or PSA level prior to surgery and the pathological stage of tumours.

\section{Discussion}

To the best of our knowledge, the present study is the first to evaluate RhoA expression not only in the centre of tumours, but also at the tumour front and in peritumoural tissues. The current study identified significantly increased RhoA expression at the tumour front compared with the tumour centre.

RhoA has previously been reported to be involved in prostate cancer invasion. Hodge et al reported increased expression of RhoA in highly invasive variants of PC-3 prostate cancer cells compared with minimally invasive variants (23). Neuropeptide-stimulated migration in prostate-cancer cells has been revealed to be mediated by RhoA (24). RhoA has also been reported to induce migration towards monocyte chemoattractant protein 1 in PC-3 cells (25). Several inhibitors of the migration of prostate cancer cells, such as transmembrane protein with epidermal growth factor-like and two follistatin-like domains 2 (26), microRNA-34a (27), exchange protein directly activated by cyclic adenosine monophosphate (28), FTY720 (29) and WIN55,21-2 (30), have been reported to inhibit RhoA activity in prostate cancer cell lines. 
The microenvironment of a tumour is highly complex and varies between locations (31). Several studies have addressed this issue and demonstrated that the tumour front and tumour centre exhibit different characteristics, resulting in different behaviours $(32,33)$. In colorectal carcinomas, Cianchi et al revealed that cells at the invasive front exhibit more aggressive behaviour compared to cells within the central regions of tumours (32).

The present study indicates that prostate cancer cells at the tumour front demonstrate a more aggressive phenotype, and express specific and different features compared with cells at the centre of the tumour. Chemokine (C-X-C motif) receptor 4 (CXCR4), which is implicated in tumour invasion through the extracellular matrix, is specifically expressed at the tumour front of prostate tumours, whereas the expression of CXCR4 at the centre of tumours is low (33). The present results indicate that RhoA expression in the centre of the tumour is low. The current findings also clearly highlight the importance of investigating the tumour front and the surrounding peritumoural tissue prior to undertaking large assays, to avoid biased interpretations.

As RhoA is implicated in cancer-cell invasion, it was hypothesized that cancer cells expressing RhoA at the tumour front may demonstrate increased mobility and aggressiveness (10). This hypothesis is also supported by the present finding that the probability of PSA relapse subsequent to surgery was increased in patients with high RhoA expression at tumour fronts, although this was not statistically significant. The present results also reveal that high RhoA expression was associated with high-grade prostate cancer in the tumour centre and the tumour front, indicating that poorly differentiated tumours were more likely to express RhoA, which may also facilitate tissue invasion.

Although a previous study by Schmidt et al has suggested that the expression of RhoA in benign prostate glands is decreased (19), the present study revealed opposite findings. In the study by Schmidt et al, the authors used biopsy material from 91 patients with localised prostate cancer. This study observed decreased expression of RhoA in benign regions compared with cancerous regions. However, the true location of these benign regions may not be accurately identified on biopsy materials. The benign regions may have been located close to tumour foci that were not sampled by the biopsy. It is also challenging to determine whether the tumoural tissue sampled by the biopsy was located at the tumour front or the centre. Notably, significantly increased RhoA expression was identified in the distant peritumoural region, whereas the activity of RhoA, as determined by G-LISA, was decreased in these regions compared with tumoural areas. As RhoA is a ubiquitous protein with numerous functions, the present results may indicate that the expression and activity of RhoA in the distant peritumoural region was not associated with the presence of cancer.

RhoA is also involved in secretory granule trafficking and exocytosis $(34,35)$, and may execute these functions in the epithelial cells of distant tissues. Although RhoA expression was decreased in the tumour cells, the increased activity of RhoA may consolidate the hypothesis that RhoA performs a specific role in prostate cancer progression. As RhoA targets several downstream effectors, with Rho-associated, coiled-coil containing protein kinase 1 and 2 being the most important, a comparison between the specific activities of these effectors within the various tissue locations may improve the present understanding of the RhoA pathways and the implications of these pathways on prostate cancer progression.

The present study has several limitations, the most important being the small sample size, which may have biased the statistical analysis. A larger cohort is required to reliably test the association between RhoA expression and other adverse clinical and pathological features. Another limitation of the current study is the absence of whole gland analysis. Only the expression in the index tumour was focused on, and contiguous or distant tumours may not have exhibited the same profile of RhoA expression. Additional analyses should be conducted on more samples to assess the downstream effectors for the whole prostatic gland.

In conclusion, the present study has identified increased RhoA expression in prostate tumour fronts and has determined associations between increased RhoA expression and cancer relapse, increased RhoA expression and increased Gleason score. This indicates an association between RhoA expression and aggressiveness of the prostate cancer. The present findings may provide a foundation for novel therapeutic approaches that may inhibit the clinical aggressiveness of prostate cancer.

\section{Acknowledgements}

The authors would like to thank Dr T. Hua-Huy (Service de Physiologie, Cochin Hospital, Paris, France) for aiding with the present study and comments on the manuscript. This study was supported by the Program of International Science \& Technology Cooperation (grant no. 2012DFG31440), awarded by the Ministry of Science and Technology, People's Republic of China. The authors thank NewMed Publishing Services for providing pro bono final editing services.

\section{References}

1. Siegel R, Naishadham D and Jemal A: Cancer statistics, 2013. CA Cancer J Clin 63: 11-30, 2013.

2. Haas GP, Delongchamps NB, Jones RF, Chandan V, Serio AM, Vickers AJ, Jumbelic M, Threatte G, Korets R, Lilja H and de la Roza G: Needle biopsies on autopsy prostates: Sensitivity of cancer detection based on true prevalence. J Natl Cancer Inst 99: 1484-1489, 2007.

3. Breslow N, Chan CW, Dhom G, Drury RA, Franks LM, Gellei B, Lee YS, Lundberg S, Sparke B, Sternby NH and Tulinius H: Latent carcinoma of prostate at autopsy in seven areas. The International Agency for Research on Cancer, Lyons, France. Int J Cancer 20: 680-688, 1977.

4. Thompson IM: Latent carcinoma of the prostate. Eur Urol 39 (Suppl 4): S41-S42, 2001

5. Stamey TA, Yemoto CM, McNeal JE, Sigal BM and Johnstone IM: Prostate cancer is highly predictable: A prognostic equation based on all morphological variables in radical prostatectomy specimens. J Urol 163: 1155-1160, 2000.

6. Gulati R, Wever EM, Tsodikov A, Penson DF, Inoue LY, Katcher J, Lee SY, Heijnsdijk EA, Draisma G, de Koning HJ and Etzioni R: What if i don't treat my PSA-detected prostate cancer? Answers from three natural history models. Cancer Epidemiol Biomarkers Prev 20: 740-750, 2011.

7. Putzke AP, Ventura AP, Bailey AM, Akture C, Opoku-Ansah J, Celiktaş M, Hwang MS, Darling DS, Coleman IM, Nelson PS, et al: Metastatic progression of prostate cancer and e-cadherin regulation by zebl and SRC family kinases. Am J Pathol 179: 400-410, 2011.

8. Tomita K, van Bokhoven A, van Leenders GJ, Ruijter ET, Jansen CF, Bussemakers MJ and Schalken JA: Cadherin switching in human prostate cancer progression. Cancer Res 60: 3650-3654, 2000. 
9. Gotzmann J, Mikula M, Eger A, Schulte-Hermann R, Foisner R, Beug H and Mikulits W: Molecular aspects of epithelial cell plasticity: Implications for local tumor invasion and metastasis. Mutat Res 566: 9-20, 2004.

10. Pertz O, Hodgson L, Klemke RL and Hahn KM: Spatiotemporal dynamics of RhoA activity in migrating cells. Nature 440 1069-1072, 2006.

11. Machacek M, Hodgson L, Welch C, Elliott H, Pertz O, Nalbant P, Abell A, Johnson GL, Hahn KM and Danuser G: Coordination of Rho GTPase activities during cell protrusion. Nature 461: 99-103, 2009.

12. Kurokawa K and Matsuda M: Localized RhoA activation as a requirement for the induction of membrane ruffling. Mol Biol Cell 16: 4294-4303, 2005

13. Sakurai-Yageta M, Recchi C, Le Dez G, Sibarita JB, Daviet L, Camonis J, D'Souza-Schorey C and Chavrier P: The interaction of IQGAP1 with the exocyst complex is required for tumor cell invasion downstream of Cde42 and RhoA. J Cell Biol 181: 985-998, 2008

14. Hotary K, Li XY, Allen E, Stevens SL and Weiss SJ: A cancer cell metalloprotease triad regulates the basement membrane transmigration program. Genes Dev 20: 2673-2686, 2006.

15. Berton S, Belletti B, Wolf K, Canzonieri V, Lovat F, Vecchione A, Colombatti A, Friedl P and Baldassarre G: The tumor suppressor functions of $\mathrm{p} 27(\mathrm{kip} 1)$ include control of the mesenchymal/amoeboid transition. Mol Cell Biol 29: 5031-5045, 2009.

16. Horiuchi A, Imai T, Wang C, Ohira S, Feng Y, Nikaido T and Konishi I: Up-regulation of small GTPases, RhoA and RhoC, is associated with tumor progression in ovarian carcinoma. Lab Invest 83: 861-870, 2003.

17. Modolo F, Biz MT, de Sousa SM, Fachinelli Rde L and Crema VO: Immunohistochemical expression of Rho GTPases in ameloblastomas. J Oral Pathol Med 41: 400-407, 2012.

18. Fritz G, Brachetti C, Bahlmann F, Schmidt M and Kaina B: Rho GTPases in human breast tumours: Expression and mutation analyses and correlation with clinical parameters. Br J Cancer 87: 635-644, 2002

19. Schmidt LJ, Duncan K, Yadav N, Regan KM, Verone AR, Lohse CM, Pop EA, Attwood K, Wilding G, Mohler JL, et al RhoA as a mediator of clinically relevant androgen action in prostate cancer cells. Mol Endocrinol 26: 716-735, 2012.

20. Epstein JI, Allsbrook WC Jr, Amin MB and Egevad LL; ISUP Grading Committee: The 2005 International Society of Urological Pathology (ISUP) Consensus Conference on Gleason Grading of Prostatic Carcinoma. Am J Surg Pathol 29: 1228-1242, 2005.

21. Sobin LH, Gospodarowicz MK and Wittekind C (eds): Prostate. In: TNM Classification of Malignant Tumors. 7th edition. John Wiley \& Sons, Hoboken, NJ, pp243-248, 2009.

22. Taylor CR and Levenson RM: Quantification of immunohistochemistry - issues concerning methods, utility and semiquantitative assessment II. Histopathology 49: 411-424, 2006.
23. Hodge JC, Bub J, Kaul S, Kajdacsy-Balla A and Lindholm PF: Requirement of RhoA activity for increased nuclear factor kappaB activity and PC-3 human prostate cancer cell invasion. Cancer Res 63: 1359-1364, 2003.

24. Zheng R, Iwase A, Shen R, Goodman OB Jr, Sugimoto N, Takuwa Y, Lerner DJ and Nanus DM: Neuropeptide-stimulated cell migration in prostate cancer cells is mediated by RhoA kinase signaling and inhibited by neutral endopeptidase. Oncogene 25: 5942-5952, 2006

25. Loberg RD, Tantivejkul K, Craig M, Neeley CK and Pienta KJ: PAR1-mediated RhoA activation facilitates CCL2-induced chemotaxis in PC-3 cells. J Cell Biochem 101: 1292-1300, 2007.

26. Chen X, Corbin JM, Tipton GJ, Yang LV, Asch AS and Ruiz-Echevarría MJ: The TMEFF2 tumor suppressor modulates integrin expression, RhoA activation and migration of prostate cancer cells. Biochim Biophys Acta 1843: 1216-1224, 2014.

27. Yamamura S, Saini S, Majid S, Hirata H, Ueno K, Deng G and Dahiya R: MicroRNA-34a modulates c-Myc transcriptional complexes to suppress malignancy in human prostate cancer cells. PLoS One 7: e29722, 2012.

28. Grandoch M, Rose A, ter Braak M, Jendrossek V, Rübben H, Fischer JW, Schmidt M and Weber AA: Epac inhibits migration and proliferation of human prostate carcinoma cells. $\mathrm{Br}$ J Cancer 101: 2038-2042, 2009.

29. Zhou C, Ling MT, Kin-Wah Lee T, Man K, Wang $X$ and Wong YC: FTY720, a fungus metabolite, inhibits invasion ability of androgen-independent prostate cancer cells through inactivation of RhoA-GTPase. Cancer Lett 233: 36-47, 2006.

30. Nithipatikom K, Gomez-Granados AD, Tang AT, Pfeiffer AW, Williams CL and Campbell WB: Cannabinoid receptor type 1 (CB1) activation inhibits small GTPase RhoA activity and regulates motility of prostate carcinoma cells. Endocrinology 153: 29-41, 2012.

31. Joyce JA: Therapeutic targeting of the tumor microenvironment. Cancer Cell 7: 513-520, 2005.

32. Cianchi F, Cuzzocrea S, Vinci MC, Messerini L, Comin CE, Navarra G, Perigli G, Centorrino T, Marzocco S, Lenzi E, et al: Heterogeneous expression of cyclooxygenase-2 and inducible nitric oxide synthase within colorectal tumors: Correlation with tumor angiogenesis. Dig Liver Dis 42: 20-27, 2010.

33. Delongchamps NB, Beuvon F, Mathieu JR, Delmas S, Metzger I, Prats H and Cabon F: CXCR4 is highly expressed at the tumor front but not in the center of prostate cancers. World J Urol 33: 281-287, 2015.

34. Gasman S, Chasserot-Golaz S, Hubert P, Aunis D and Bader MF Identification of a potential effector pathway for the trimeric Go protein associated with secretory granules. Go stimulates a granule-bound phosphatidylinositol 4-kinase by activating RhoA in chromaffin cells. J Biol Chem 273: 16913-16920, 1998.

35. Frantz C, Coppola T and Regazzi R: Involvement of Rho GTPases and their effectors in the secretory process of PC12 cells. Exp Cell Res 273: 119-126, 2002. 